\title{
The Impact of Endogenous Estrogen Exposures on the Characteristics and Outcomes of Estrogen Receptor Positive, Early-stage Breast Cancer
}

\author{
Yasmin Korzets \\ Tel Aviv Sourasky Medical Center \\ Orly Yariv \\ Rabin Medical Center \\ Raz Mutai \\ Rabin Medical Center \\ Assaf Moore \\ Rabin Medical Center \\ Tzippy Shochat \\ Rabin Medical Center \\ Rinat yerushalmi \\ Rabin Medical Center \\ Hadar Goldvaser ( $\boldsymbol{\sim}$ hadar7g@gmail.com ) \\ Shaare Zedek Medical Center, The Integrated Oncology Institute
}

Research Article

Keywords: breast cancer, estrogen, parity, menopause, menarche

Posted Date: June 17th, 2021

DOI: https://doi.org/10.21203/rs.3.rs-618743/v1

License: (ㄷ) This work is licensed under a Creative Commons Attribution 4.0 International License. Read Full License 


\section{Abstract}

Background: Menstrual and parity history might impact the risk for breast cancer. Data on the impact of these factors on other tumor characteristics are limited.

Methods: A single center retrospective cohort study comprising all women with estrogen receptor (ER) positive, human epidermal growth factor receptor 2 (HER2) negative, early breast cancer whose tumors were sent to OncotypeDX analysis. The impact on tumor characteristics including Oncotype recurrence score and on outcomes was evaluated, by prespecified subgroups, including: age of menarche ( $<12$ vs. $\geq 12$ years), number of deliveries ( 0 vs. $\geq 1$ childbirth and $\geq 5$ childbirth vs. other), age of first delivery ( $\geq 30$ years vs. younger age) and postmenopausal compared to premenopausal. The impact of age of menopause was also assessed categorically, using early ( $<45$ years) and late age of menopause ( $>55$ years).

Results: A total of 620 women were included. After median follow-up of 10.4 years, early menopause was associated with significantly worse disease-free survival $(H R=2.26, p=0.004)$ and overall-survival $(H R=2.60, p=0.004)$, and multiparity was associated with significant worse diseasefree survival $(H R=2.16, p=0.026)$. These differences remain significant in multivariate analyses. Post-menopausal women were more likely to have stronger ER intensity $(p=0.002)$ but progesterone receptor $(P R)$ positivity was less frequent $(p=0.009$. Early age of menarche was associated with PR positivity $(p=0.039)$. No other associations were found between the evaluated subgroups and tumor characteristics.

Conclusions: The impact of endogenous estrogen exposure had little effect on breast cancer characteristics of early stage, luminal disease. Early menopause and multiparity were associated with worse outcome.

\section{Introduction}

Breast cancer accounts for $30 \%$ of all cancer diagnosed in women, with a lifetime risk of $12.4 \%$ [1]. Estrogen and its metabolites have a role in breast cancer development. Exposure to high levels of either endogenous or exogenous estrogen increase the risk of breast cancer, particularly estrogen receptor (ER) positive breast cancer [2], which is overexpressed in approximately $75 \%$ of all breast cancers [3].

Excess risk of breast cancer has been attributed to early menarche and late menopause, this is associated to lengthening woman's reproductive years [4]. Compared to early menarche, late menarche is associated with decreased risk for breast cancer which is more robust for ER positive disease than ER negative disease [5]. Late menopause independently increases the risk for breast cancer, with a relative increase of $3 \%$ for every year older at menopause [4].

Parity also effects breast cancer risk through hormonal mechanisms. Nulliparity is related to an increase risk of breast cancer, whereas multiparity has an overall protective effect, although there is an increased risk for breast cancer during the first two decades after childbirth and then it gradually decreases $[6,7]$. Age at first full-term pregnancy is another factor related to breast cancer risk, with lower breast cancer risk in women with younger age of first birth [8]. Obesity is a known risk factor for cancer, higher BMI increases the risk for ER positive breast cancer specifically in postmenopausal women, possibly explained by higher estrogen levels resulting from the peripheral conversion of estrogen precursors from adipose tissue $[9,10]$.

The impact of endogenous estrogen on breast cancer characteristics is less known. Earlier age at menarche and older age of menopause are associated with more frequently lobular tumors [4]. Older age at menopause is associated with the development of ER positive tumors rather than to ER negative tumors [4]. An association between the baseline levels of sex-hormone has also been described in postmenopausal women, with higher level of estradiol and testosterone associated with increased risk of ER positive disease, but not with ER negative breast cancer [11, 12].

Breast cancer is a diversified disease, with different histopathological characteristics and molecular subtypes that determine both treatment and prognosis [13]. ER positive, human epidermal growth factor receptor 2 (HER2) negative-subtype represents approximately 65-70\% of invasive breast cancer and compared to other subtypes have a better prognosis [13]. Yet, the luminal-subtype has a spectrum of variables affecting its prognosis and treatment, including the extent of the disease, grade, ki67, intensity of ER and genomic risk [14-17]. One of the genomic assays used in this population is the 21-gene recurrence-score assay (Oncotype DX, genomic Health) which provides both predictive and prognostic information in early stage luminal disease [18].

The aim of our study was to investigate the effect of endogenous estrogen on histological tumor characteristics and on the genomic risk in early breast cancer women with ER positive, HER2 negative disease and to explore the correlation of these exposures on outcomes.

\section{Methods}

This was a retrospective single center cohort study. All women who were treated in our institute between 2005 and 2012 with ER positive, HER2 negative, early breast cancer whose tumors were sent to Oncotype DX analysis were included. The Clalit Heath Services are the medical provider for the majority of the included patients in this cohort. 
A detailed review of patients' medical records and pathological reports was conducted. Data on demographics and pre-specified clinicalpathological parameters were extracted including: tumor size (categorized as $\mathrm{T} \leq 1 \mathrm{~cm}, 1<\mathrm{T} \leq 2 \mathrm{~cm}$ and $\mathrm{T}>2 \mathrm{~cm}$ ), nodal status (negative or positive, including both macro- and micrometastases), intensity of ER and progesterone receptor (PR) expression, grade, ki67, lymphovascular and perineural invasion and Oncotype DX recurrence score (RS). RS was categorized by the TailorX groups: low risk: RS $\leq 25$ and high risk: RS > 25 [20].

Data on endogenous estrogen exposure and parity were collected including: age of menarche, number of childbirth and age of first delivery, menopausal status and age of menopause. Data on any loco-regional, distant recurrence and death were also recorded. Data lock was in $18 / 06 / 2020$. Time of recurrence was defined as the date of biopsy from site of recurrence or the date of abnormal imaging suggestive for metastatic disease. Disease-free survival (DFS) was defined as the time between breast surgery to an event (recurrence or death) or data lock. Duration of survival was defined as time from initial breast cancer diagnosis to date of death or time of data lock. Patients' vital status was ascertained through Israel's ministry of interior database.

The impact of endogenous estrogen exposure and parity on breast cancer characteristics and on outcomes was evaluated. Comparisons were conducted for the pre-specified subgroups including: age of menarche (age < 12 compared to age $\geq 12$ ), nulliparity (yes compared to no), multiparity (women with 5 or more childbirths compared to less than 5 childbirths) and menopause (postmenopausal compared to premenopausal). For postmenopausal women comparisons were done for early menopause (age $<45$ compared to menopause age $\geq 45$ ) and late menopause (age > 55 compared to menopause at the age $\leq 55$ ). As data were analyzed anonymously, no consent was required. The study protocol was approved by our institutional ethics committee.

\section{Statistical analysis}

Statistical analysis was generated using SAS Software, Version 9.4. Data were reported descriptively by each of the pre-specified categories as described above. Categorical variables were presented by as proportions and continuous variables were presented by mean and standard deviation (SD) or Median (Range) as appropriate. T-test for normally distributed variables or Mann Whintney for non normal were used to compare the value of continuous variables between study groups. Fisher's exact test was used to compare the value of categorical variables between study groups.

The impact of age of menarche, menopause, age of menopause and parity on DFS and OS were assessed. Overall-survival (OS) and DFS were assessed by Kaplan-Meier survival analysis, with the log-rank test. For significant differences in outcomes in univariate analyses, multivariate analysis for age, tumor size, nodal status, grade and Oncotype RS were assessed by the Cox proportional hazards model. Two-sided p-values less than 0.05 were considered statistically significant.

\section{Results}

We identified 705 patients with early breast cancer whose tumor were sent to ODX analysis at our institution from 2005 to 2012 . 85 patients were excluded, remaining 620 women who were included in the study cohort.

Median age was 61 years (range 34-85 years), 75\% (464) women were postmenopausal, most of them $86 \%$ menopaused before the age of 55 and $14 \%$ had menopause before the age of 45 . Median age of menarche was 13 years and in $86 \%$ age of menarche was 12 and above. Median number of deliveries was 3 (range $0-14$ ), $6 \%$ were multiparous and $10 \%$ were nullipara. Invasive ductal carcinoma (IDC) was the most common histology (81\%), followed by invasive lobular carcinoma (ILC) (12\%). Women were most likely to present with tumor size $\leq 2 \mathrm{~cm}$ (77\%) and node negative disease (82\%). Grade was well, moderately and poorly differentiated in $17 \%, 66 \%$ and $17 \%$ of tumors, respectively with strong ER intensity in $76 \%$ of women and negative PR in $14 \%$ of women. Ki67 was $20 \%$ or lower in $78 \%$ of tumors. ODX RS was 25 or lower in $82 \%$. Perineural and angiolymphatic invasion were uncommon presenting in $9 \%$ and $6 \%$ of tumors, respectively. Overall, $98 \%$ women were treated with adjuvant endocrine therapy and of $77 \%$ of women with high genomic risk (RS $>25$ ) were treated with adjuvant chemotherapy.

Histo-pathological characteristics by the pre-specified subgroups are presented in Table 1. Post-menopausal women were more likely to have stronger ER intensity (79\% vs. 65\%, p = 0.002), but PR negativity was significantly more frequent $(16 \%$ vs. $7 \%, p=0.009)$. Early age of menarche was associated with PR positivity ( $94 \%$ vs. $85 \%, p=0.039)$. No other associations were found between endogenous estrogen exposures and tumor characteristics. 
Table 1

The impact of endogenic estrogen exposure of tumor characteristics.

\begin{tabular}{|c|c|c|c|c|c|c|c|c|c|c|c|c|c|c|}
\hline \multirow[t]{2}{*}{$\mathbf{N}(\%)$} & \multicolumn{2}{|c|}{$\begin{array}{l}\text { Age of } \\
\text { menarche }\end{array}$} & \multicolumn{2}{|c|}{ Nulliparity } & \multicolumn{2}{|c|}{$\begin{array}{l}\text { Multiparity ( } \geq \\
5 \text { deliveries }\end{array}$} & \multicolumn{2}{|c|}{$\begin{array}{l}\text { Age at first } \\
\text { delivery }\end{array}$} & \multicolumn{2}{|c|}{ Menopause } & \multicolumn{2}{|c|}{$\begin{array}{l}\text { Early } \\
\text { menopause } \\
(\text { age < 45) }\end{array}$} & \multicolumn{2}{|c|}{$\begin{array}{l}\text { Late menopause } \\
(\text { age }>55)\end{array}$} \\
\hline & $\begin{array}{l}<12 \\
(n= \\
69)\end{array}$ & $\begin{array}{l}\geq 12 \\
(n= \\
448)\end{array}$ & $\begin{array}{l}\text { Yes } \\
(n= \\
62)\end{array}$ & $\begin{array}{l}\text { No } \\
(n= \\
543)\end{array}$ & $\begin{array}{l}\text { No } \\
(n= \\
569)\end{array}$ & $\begin{array}{l}\text { Yes } \\
(n= \\
36)\end{array}$ & $\begin{array}{l}<30 \\
(n= \\
284)\end{array}$ & $\begin{array}{l}\geq 30 \\
(n= \\
58)\end{array}$ & $\begin{array}{l}\text { No } \\
(n= \\
131)\end{array}$ & $\begin{array}{l}\text { Yes } \\
(n= \\
464)\end{array}$ & $\begin{array}{l}\text { Yes } \\
(n= \\
65)\end{array}$ & $\begin{array}{l}\text { No } \\
(n= \\
353)\end{array}$ & $\begin{array}{l}\text { No } \\
(n= \\
401)\end{array}$ & $\begin{array}{l}\text { Yes } \\
(n=17)\end{array}$ \\
\hline $\begin{array}{l}T \text { size } \\
T<1 \mathrm{~cm}\end{array}$ & $\begin{array}{l}12 \\
(17 \%)\end{array}$ & $\begin{array}{l}98 \\
(22 \%)\end{array}$ & $\begin{array}{l}16 \\
(26 \%)\end{array}$ & $\begin{array}{l}117 \\
(21 \%)\end{array}$ & $\begin{array}{l}128 \\
(23 \%)\end{array}$ & $\begin{array}{l}5 \\
(14 \%)\end{array}$ & $\begin{array}{l}55 \\
(19 \%)\end{array}$ & $\begin{array}{l}18 \\
(31 \%)\end{array}$ & $\begin{array}{l}27 \\
(21 \%)\end{array}$ & $\begin{array}{l}106 \\
(23 \%)\end{array}$ & $\begin{array}{l}15 \\
(23 \%)\end{array}$ & $\begin{array}{l}81 \\
(23 \%)\end{array}$ & 92 & $\begin{array}{l}4 \\
(23.5 \%)\end{array}$ \\
\hline $1<\mathrm{T} \leq 2$ & $\begin{array}{l}39 \\
(57 \%)\end{array}$ & $\begin{array}{l}244 \\
(55 \%)\end{array}$ & $\begin{array}{l}33 \\
(53 \%)\end{array}$ & $\begin{array}{l}297 \\
(55 \%)\end{array}$ & $\begin{array}{l}307 \\
(54 \%)\end{array}$ & $\begin{array}{l}23 \\
(64 \%)\end{array}$ & $\begin{array}{l}162 \\
(57 \%)\end{array}$ & $\begin{array}{l}32 \\
(55 \%)\end{array}$ & $\begin{array}{l}66 \\
(50 \%)\end{array}$ & $\begin{array}{l}259 \\
(56 \%)\end{array}$ & $\begin{array}{l}34 \\
(52 \%)\end{array}$ & $\begin{array}{l}198 \\
(56 \%)\end{array}$ & 223 & 9 \\
\hline $\mathrm{T}>2 \mathrm{~cm}$ & $\begin{array}{l}18 \\
(26 \%)\end{array}$ & $\begin{array}{l}105 \\
(23 \%)\end{array}$ & $\begin{array}{l}13 \\
(21 \%)\end{array}$ & $\begin{array}{l}128 \\
(24 \%)\end{array}$ & $\begin{array}{l}133 \\
(23 \%)\end{array}$ & $\begin{array}{l}8 \\
(22 \%)\end{array}$ & $\begin{array}{l}67 \\
(24 \%)\end{array}$ & $\begin{array}{l}8 \\
(14 \%)\end{array}$ & $\begin{array}{l}38 \\
(29 \%)\end{array}$ & $\begin{array}{l}98 \\
(21 \%)\end{array}$ & $\begin{array}{l}16 \\
(25 \%)\end{array}$ & $\begin{array}{l}73 \\
(21 \%)\end{array}$ & $\begin{array}{l}85 \\
(21 \%)\end{array}$ & $\begin{array}{l}4 \\
(23.5 \%)\end{array}$ \\
\hline $\begin{array}{l}\text { P value for } \\
\text { the } \\
\text { difference } \\
\text { (T) }\end{array}$ & 0.675 & & 0.725 & & 0.413 & & 0.074 & & 0.169 & & 0.763 & & 0.968 & \\
\hline $\begin{array}{l}\text { Nodes } \\
\text { Negative }^{\mathrm{a}}\end{array}$ & $\begin{array}{l}54 \\
(79 \%)\end{array}$ & $\begin{array}{l}374 \\
(84 \%)\end{array}$ & $\begin{array}{l}53 \\
(85 \%)\end{array}$ & $\begin{array}{l}448 \\
(83 \%)\end{array}$ & $\begin{array}{l}472 \\
(83 \%)\end{array}$ & $\begin{array}{l}29 \\
(81 \%)\end{array}$ & $\begin{array}{l}233 \\
(83 \%)\end{array}$ & $\begin{array}{l}48 \\
(83 \%)\end{array}$ & $\begin{array}{l}105 \\
(80 \%)\end{array}$ & $\begin{array}{l}386 \\
(84 \%)\end{array}$ & $\begin{array}{l}54 \\
(84 \%)\end{array}$ & $\begin{array}{l}293 \\
(84 \%)\end{array}$ & $\begin{array}{l}333 \\
(84 \%)\end{array}$ & $\begin{array}{l}14 \\
(82 \%)\end{array}$ \\
\hline $\begin{array}{l}\text { P value for } \\
\text { the } \\
\text { difference } \\
\text { (N) }\end{array}$ & 0.383 & & 0.721 & & 0.647 & & 1.000 & & 0.355 & & 1.000 & & 0.745 & \\
\hline $\begin{array}{l}\text { Histology } \\
\text { subtype }^{c}\end{array}$ & $\begin{array}{l}54 \\
(79 \%)\end{array}$ & $\begin{array}{l}360 \\
(80 \%)\end{array}$ & $\begin{array}{l}49 \\
(79 \%)\end{array}$ & $\begin{array}{l}440 \\
(81 \%)\end{array}$ & $\begin{array}{l}462 \\
(81 \%)\end{array}$ & $\begin{array}{l}27 \\
(75 \%)\end{array}$ & $\begin{array}{l}227 \\
(80 \%)\end{array}$ & $\begin{array}{l}45 \\
(78 \%)\end{array}$ & $\begin{array}{l}106 \\
(81 \%)\end{array}$ & $\begin{array}{l}373 \\
(81 \%)\end{array}$ & $\begin{array}{l}54 \\
(83 \%)\end{array}$ & $\begin{array}{l}281 \\
(80 \%)\end{array}$ & $\begin{array}{l}320 \\
(80 \%)\end{array}$ & $\begin{array}{l}15 \\
(88 \%)\end{array}$ \\
\hline & $\begin{array}{l}13 \\
(19 \%)\end{array}$ & $\begin{array}{l}53 \\
(12 \%)\end{array}$ & $\begin{array}{l}7 \\
(11 \%)\end{array}$ & $\begin{array}{l}68 \\
(13 \%)\end{array}$ & $\begin{array}{l}69 \\
(12 \%)\end{array}$ & $\begin{array}{l}6 \\
(17 \%)\end{array}$ & $\begin{array}{l}40 \\
(14 \%)\end{array}$ & $\begin{array}{l}11 \\
(19 \%)\end{array}$ & $\begin{array}{l}15 \\
(11 \%)\end{array}$ & $\begin{array}{l}60 \\
(13 \%)\end{array}$ & 8 & $\begin{array}{l}46 \\
(13 \%)\end{array}$ & $\begin{array}{l}53 \\
(13 \%)\end{array}$ & $\begin{array}{l}1 \\
(6 \%)\end{array}$ \\
\hline Other ${ }^{b}$ & $\begin{array}{l}2 \\
(3 \%)\end{array}$ & $\begin{array}{l}35 \\
(8 \%)\end{array}$ & $\begin{array}{l}6 \\
(10 \%)\end{array}$ & $\begin{array}{l}34 \\
(6 \%)\end{array}$ & $\begin{array}{l}37 \\
(7 \%)\end{array}$ & $\begin{array}{l}3 \\
(8 \%)\end{array}$ & $\begin{array}{l}17 \\
(6 \%)\end{array}$ & $\begin{array}{l}2 \\
(3 \%)\end{array}$ & $\begin{array}{l}10 \\
(8 \%)\end{array}$ & $\begin{array}{l}30 \\
(6 \%)\end{array}$ & $\begin{array}{l}3 \\
(5 \%)\end{array}$ & $\begin{array}{l}26 \\
(7 \%)\end{array}$ & $\begin{array}{l}28 \\
(7 \%)\end{array}$ & $\begin{array}{l}1 \\
(6 \%)\end{array}$ \\
\hline $\begin{array}{l}\text { P value for } \\
\text { the } \\
\text { difference } \\
\text { (histological } \\
\text { subtype) }\end{array}$ & 0.114 & & 0.584 & & 0.640 & & 0.506 & & 0.824 & & 0.704 & & 0.653 & \\
\hline Grade & $\begin{array}{l}6 \\
(11 \%)\end{array}$ & $\begin{array}{l}65 \\
(18 \%)\end{array}$ & $\begin{array}{l}11 \\
(21 \%)\end{array}$ & $\begin{array}{l}74 \\
(17 \%)\end{array}$ & $\begin{array}{l}80 \\
(17 \%)\end{array}$ & $\begin{array}{l}5 \\
(19 \%)\end{array}$ & $\begin{array}{l}38 \\
(16 \%)\end{array}$ & $\begin{array}{l}9 \\
(20 \%)\end{array}$ & $\begin{array}{l}24 \\
(22 \%)\end{array}$ & $\begin{array}{l}59 \\
(16 \%)\end{array}$ & $\begin{array}{l}9 \\
(17 \%)\end{array}$ & $\begin{array}{l}45 \\
(16 \%)\end{array}$ & $\begin{array}{l}51 \\
(16 \%)\end{array}$ & $\begin{array}{l}3 \\
(19 \%)\end{array}$ \\
\hline 2 & $\begin{array}{l}37 \\
(67 \%)\end{array}$ & $\begin{array}{l}243 \\
(66 \%)\end{array}$ & $\begin{array}{l}35 \\
(67 \%)\end{array}$ & $\begin{array}{l}291 \\
(65 \%)\end{array}$ & $\begin{array}{l}308 \\
(65 \%)\end{array}$ & $\begin{array}{l}18 \\
(69 \%)\end{array}$ & $\begin{array}{l}152 \\
(66 \%)\end{array}$ & $\begin{array}{l}30 \\
(65 \%)\end{array}$ & $\begin{array}{l}67 \\
(61 \%)\end{array}$ & $\begin{array}{l}254 \\
(66 \%)\end{array}$ & $\begin{array}{l}37 \\
(68 \%)\end{array}$ & $\begin{array}{l}188 \\
(66 \%)\end{array}$ & $\begin{array}{l}215 \\
(66 \%)\end{array}$ & $\begin{array}{l}10 \\
(62 \%)\end{array}$ \\
\hline 3 & $\begin{array}{l}12 \\
(22 \%)\end{array}$ & $\begin{array}{l}61 \\
(16 \%)\end{array}$ & $\begin{array}{l}6 \\
(12 \%)\end{array}$ & $\begin{array}{l}82 \\
(18 \%)\end{array}$ & $\begin{array}{l}85 \\
(18 \%)\end{array}$ & $\begin{array}{l}3 \\
(12 \%)\end{array}$ & $\begin{array}{l}42 \\
(18 \%)\end{array}$ & $\begin{array}{l}7 \\
(15 \%)\end{array}$ & $\begin{array}{l}18 \\
(17 \%)\end{array}$ & $\begin{array}{l}67 \\
(18 \%)\end{array}$ & $\begin{array}{l}8 \\
(15 \%)\end{array}$ & $\begin{array}{l}53 \\
(18 \%)\end{array}$ & $\begin{array}{l}58 \\
(18 \%)\end{array}$ & 3 (19\%) \\
\hline
\end{tabular}

\footnotetext{
a Node positive included micro-metastasis and marco-metastasis to lymph nodes.

b Other subtypes include mixed ductal-lobular

${ }^{\mathrm{c}}$ Other histology subtypes included medullary, mucinous, papillary and tubular carcinomas.

Abbreviations:

ER-estrogen receptor, IDC- invasive ductal carcinoma, ILC- invasive lobular carcinoma, OC- Oral Contraceptive, PNI- perineural invasion, PRprogesterone receptor, LVI- lymphovascular invasion. RS- recurrence score,

Data were not available for: age of menarche- $n=103$, number of deliveries- $n=15$, age of first delivery- $n=278$, menopause- $n=25$, age of menopause- $\mathrm{n}=202$.
} 


\begin{tabular}{|c|c|c|c|c|c|c|c|c|c|c|c|c|c|c|}
\hline $\mathbf{N}(\%)$ & \multicolumn{2}{|c|}{$\begin{array}{l}\text { Age of } \\
\text { menarche }\end{array}$} & \multicolumn{2}{|c|}{ Nulliparity } & \multicolumn{2}{|c|}{$\begin{array}{l}\text { Multiparity ( } \geq \\
5 \text { deliveries }\end{array}$} & \multicolumn{2}{|c|}{$\begin{array}{l}\text { Age at first } \\
\text { delivery }\end{array}$} & \multicolumn{2}{|c|}{ Menopause } & \multicolumn{2}{|c|}{$\begin{array}{l}\text { Early } \\
\text { menopause } \\
(\text { age < 45) }\end{array}$} & \multicolumn{2}{|c|}{$\begin{array}{l}\text { Late menopause } \\
\text { (age > 55) }\end{array}$} \\
\hline $\begin{array}{l}\text { P value for } \\
\text { the } \\
\text { difference } \\
\text { (grade) }\end{array}$ & 0.510 & & 0.581 & & 0.331 & & 0.900 & & 0.444 & & 0.899 & & 0.562 & \\
\hline ER intensity & 0 & 9 & 2 & 9 & 10 & 1 & 6 & 1 & 5 & 6 & 0 & 5 & $5(1 \%)$ & 0 \\
\hline$\leq 1$ & 17 & & & & & & & & & & 10 & & 79 & 1 \\
\hline $1<\mathrm{ER}<=2$ & $(25 \%)$ & $\begin{array}{l}97 \\
(22 \%)\end{array}$ & $\begin{array}{l}18 \\
(29 \%)\end{array}$ & $\begin{array}{l}116 \\
(21 \%)\end{array}$ & $\begin{array}{l}126 \\
(22 \%)\end{array}$ & $\begin{array}{l}8 \\
(22 \%)\end{array}$ & $\begin{array}{l}64 \\
(23 \%)\end{array}$ & $\begin{array}{l}11 \\
(19 \%)\end{array}$ & $\begin{array}{l}41 \\
(31 \%)\end{array}$ & $\begin{array}{l}90 \\
(19 \%)\end{array}$ & (15\%) & $\begin{array}{l}70 \\
(20 \%)\end{array}$ & $(20 \%)$ & $(6 \%)$ \\
\hline$E R>2$ & $\begin{array}{l}52 \\
(75 \%)\end{array}$ & $\begin{array}{l}342 \\
(76 \%)\end{array}$ & $\begin{array}{l}42 \\
(68 \%)\end{array}$ & $\begin{array}{l}418 \\
(77 \%)\end{array}$ & $\begin{array}{l}433 \\
(76 \%)\end{array}$ & $\begin{array}{l}27 \\
(75 \%)\end{array}$ & $\begin{array}{l}214 \\
(75 \%)\end{array}$ & $\begin{array}{l}46 \\
(79 \%)\end{array}$ & $\begin{array}{l}85 \\
(65 \%)\end{array}$ & $\begin{array}{l}368 \\
(79 \%)\end{array}$ & $\begin{array}{l}55 \\
(85 \%)\end{array}$ & $\begin{array}{l}278 \\
(79 \%)\end{array}$ & $\begin{array}{l}317 \\
(79 \%)\end{array}$ & $\begin{array}{l}16 \\
(94 \%)\end{array}$ \\
\hline $\begin{array}{l}\text { P value for } \\
\text { the } \\
\text { difference } \\
\text { (ER) }\end{array}$ & 0.441 & & 0.240 & & 0.905 & & 0.813 & & 0.002 & & 0.422 & & 0.316 & \\
\hline $\begin{array}{l}\text { PR } \\
\text { Negative }\end{array}$ & $\begin{array}{l}4 \\
(6 \%)\end{array}$ & $\begin{array}{l}67 \\
(15 \%)\end{array}$ & $\begin{array}{l}6 \\
(10 \%)\end{array}$ & $\begin{array}{l}81 \\
(15 \%)\end{array}$ & $\begin{array}{l}83 \\
(14 \%)\end{array}$ & $\begin{array}{l}4 \\
(11 \%)\end{array}$ & $\begin{array}{l}41 \\
(14 \%)\end{array}$ & $\begin{array}{l}3 \\
(5 \%)\end{array}$ & $\begin{array}{l}9 \\
(7 \%)\end{array}$ & $\begin{array}{l}72 \\
(16 \%)\end{array}$ & $\begin{array}{l}10 \\
(15 \%)\end{array}$ & $\begin{array}{l}53 \\
(15 \%)\end{array}$ & $\begin{array}{l}61 \\
(15 \%)\end{array}$ & $\begin{array}{l}2 \\
(12 \%)\end{array}$ \\
\hline $\begin{array}{l}\text { P value for } \\
\text { the } \\
\text { difference } \\
\text { (PR) }\end{array}$ & 0.039 & & 0.340 & & 0.806 & & 0.055 & & 0.009 & & 1.000 & & 1.000 & \\
\hline $\begin{array}{l}\text { PNI } \\
\text { Yes }\end{array}$ & $\begin{array}{l}3 \\
(4 \%)\end{array}$ & $\begin{array}{l}21 \\
(5 \%)\end{array}$ & $\begin{array}{l}2 \\
(3 \%)\end{array}$ & $\begin{array}{l}23 \\
(4 \%)\end{array}$ & $\begin{array}{l}24 \\
(4 \%)\end{array}$ & $\begin{array}{l}1 \\
(3 \%)\end{array}$ & $\begin{array}{l}12 \\
(4 \%)\end{array}$ & $\begin{array}{l}0 \\
(0 \%)\end{array}$ & $\begin{array}{l}5 \\
(4 \%)\end{array}$ & $\begin{array}{l}22 \\
(5 \%)\end{array}$ & $\begin{array}{l}2 \\
(3 \%)\end{array}$ & $\begin{array}{l}18 \\
(5 \%)\end{array}$ & $\begin{array}{l}19 \\
(5 \%)\end{array}$ & $1(6 \%)$ \\
\hline $\begin{array}{l}\text { P value for } \\
\text { the } \\
\text { difference } \\
\text { (PNI) }\end{array}$ & 1.000 & & 1.000 & & 1.000 & & 0.137 & & 0.814 & & 0.751 & & 0.588 & \\
\hline $\begin{array}{l}\text { LVI } \\
\text { Yes }\end{array}$ & $\begin{array}{l}4 \\
(6 \%)\end{array}$ & $\begin{array}{l}27 \\
(6 \%)\end{array}$ & $\begin{array}{l}2 \\
(3 \%)\end{array}$ & $\begin{array}{l}31 \\
(6 \%)\end{array}$ & $\begin{array}{l}33 \\
(6 \%)\end{array}$ & $\begin{array}{l}0 \\
(0 \%)\end{array}$ & $\begin{array}{l}16 \\
(6 \%)\end{array}$ & $\begin{array}{l}3 \\
(5 \%)\end{array}$ & $\begin{array}{l}19 \\
(7 \%)\end{array}$ & $\begin{array}{l}25 \\
(5 \%)\end{array}$ & $\begin{array}{l}3 \\
(5 \%)\end{array}$ & $\begin{array}{l}21 \\
(6 \%)\end{array}$ & $\begin{array}{l}22 \\
(6 \%)\end{array}$ & $2(12 \%)$ \\
\hline $\begin{array}{l}\text { P value for } \\
\text { the } \\
\text { difference } \\
\text { (LVI) }\end{array}$ & 1.000 & & 0.563 & & 0.250 & & 1.000 & & 0.524 & & 1.000 & & 0.269 & \\
\hline $\begin{array}{l}\text { Ki67 } \\
>20 \%\end{array}$ & $\stackrel{9}{(17 \%)}$ & $\begin{array}{l}75 \\
(23 \%)\end{array}$ & $\stackrel{9}{(18 \%)}$ & $\begin{array}{l}88 \\
(22 \%)\end{array}$ & $\begin{array}{l}91 \\
(22 \%)\end{array}$ & $\begin{array}{l}6 \\
(19 \%)\end{array}$ & $\begin{array}{l}42 \\
(21 \%)\end{array}$ & $\begin{array}{l}13 \\
(32 \%)\end{array}$ & $\begin{array}{l}20 \\
(23 \%)\end{array}$ & $\begin{array}{l}75 \\
(21 \%)\end{array}$ & $\begin{array}{l}12 \\
(24 \%)\end{array}$ & $\begin{array}{l}57 \\
(21 \%)\end{array}$ & $\begin{array}{l}65 \\
(22 \%)\end{array}$ & $\begin{array}{l}4 \\
(27 \%)\end{array}$ \\
\hline $\begin{array}{l}\text { P value for } \\
\text { the } \\
\text { difference } \\
\text { (ki67) }\end{array}$ & 0.473 & & 0.588 & & 0.825 & & 0.157 & & 0.771 & & 0.707 & & 0.748 & \\
\hline $\begin{array}{l}\text { Oncotype } \\
\text { RS }\end{array}$ & $\begin{array}{l}56 \\
(81 \%)\end{array}$ & $\begin{array}{l}372 \\
(83 \%)\end{array}$ & $\begin{array}{l}54 \\
(87 \%)\end{array}$ & $\begin{array}{l}440 \\
(81 \%)\end{array}$ & $\begin{array}{l}461 \\
(81 \%)\end{array}$ & $\begin{array}{l}33 \\
(92 \%)\end{array}$ & $\begin{array}{l}236 \\
(83 \%)\end{array}$ & $\begin{array}{l}45 \\
(78 \%)\end{array}$ & $\begin{array}{l}106 \\
(81 \%)\end{array}$ & $\begin{array}{l}383 \\
(83 \%)\end{array}$ & $\begin{array}{l}54 \\
(83 \%)\end{array}$ & $\begin{array}{l}294 \\
(83 \%)\end{array}$ & $\begin{array}{l}333 \\
(83 \%)\end{array}$ & $\begin{array}{l}15 \\
(88 \%)\end{array}$ \\
\hline $\begin{array}{l}R S<=25 \\
R S>25\end{array}$ & $\begin{array}{l}13 \\
(19 \%)\end{array}$ & $\begin{array}{l}76 \\
(17 \%)\end{array}$ & $\begin{array}{l}8 \\
(13 \%)\end{array}$ & $\begin{array}{l}103 \\
(19 \%)\end{array}$ & $\begin{array}{l}108 \\
(19 \%)\end{array}$ & $\begin{array}{l}3 \\
(8 \%)\end{array}$ & $\begin{array}{l}48 \\
(17 \%)\end{array}$ & $\begin{array}{l}13 \\
(22 \%)\end{array}$ & $\begin{array}{l}25 \\
(19 \%)\end{array}$ & $\begin{array}{l}81 \\
(17 \%)\end{array}$ & $\begin{array}{l}11 \\
(17 \%)\end{array}$ & $\begin{array}{l}59 \\
(17 \%)\end{array}$ & $\begin{array}{l}68 \\
(17 \%)\end{array}$ & $\begin{array}{l}2 \\
(12 \%)\end{array}$ \\
\hline
\end{tabular}

a Node positive included micro-metastasis and marco-metastasis to lymph nodes.

b Other subtypes include mixed ductal-lobular

${ }^{c}$ Other histology subtypes included medullary, mucinous, papillary and tubular carcinomas.

Abbreviations:

ER-estrogen receptor, IDC- invasive ductal carcinoma, ILC- invasive lobular carcinoma, OC- Oral Contraceptive, PNI- perineural invasion, PRprogesterone receptor, LVI- lymphovascular invasion. RS- recurrence score,

Data were not available for: age of menarche- $n=103$, number of deliveries- $n=15$, age of first delivery- $n=278$, menopause- $n=25$, age of menopause- $\mathrm{n}=202$. 


\begin{tabular}{|c|c|c|c|c|c|c|c|}
\hline $\mathbf{N}(\%)$ & $\begin{array}{l}\text { Age of } \\
\text { menarche }\end{array}$ & Nulliparity & $\begin{array}{l}\text { Multiparity ( } \geq \\
5 \text { deliveries }\end{array}$ & $\begin{array}{l}\text { Age at first } \\
\text { delivery }\end{array}$ & Menopause & $\begin{array}{l}\text { Early } \\
\text { menopause } \\
(\text { age < 45) }\end{array}$ & $\begin{array}{l}\text { Late menopause } \\
(\text { age }>55)\end{array}$ \\
\hline $\begin{array}{l}\text { P value for } \\
\text { the } \\
\text { difference } \\
\text { (Oncotype) }\end{array}$ & 0.732 & 0.300 & 0.124 & 0.347 & 0.698 & 1.000 & 0.749 \\
\hline \multicolumn{8}{|c|}{ a Node positive included micro-metastasis and marco-metastasis to lymph nodes. } \\
\hline \multicolumn{8}{|c|}{ b Other subtypes include mixed ductal-lobular } \\
\hline \multicolumn{8}{|c|}{ c Other histology subtypes included medullary, mucinous, papillary and tubular carcinomas. } \\
\hline \multicolumn{8}{|c|}{ Abbreviations: } \\
\hline \multicolumn{8}{|c|}{$\begin{array}{l}\text { ER-estrogen receptor, IDC- invasive ductal carcinoma, ILC- invasive lobular carcinoma, OC- Oral Contraceptive, PNI- perineural invasion, PR- } \\
\text { progesterone receptor, LVl- lymphovascular invasion. RS- recurrence score, }\end{array}$} \\
\hline
\end{tabular}

The median duration of follow-up was 10.4 years. The results of DFS are presented in Table 2 and Fig. 1. Estimated 10-year DFS was significantly worse for women with early menopause (71.3\% vs. $86.3 \%), \mathrm{HR}=2.26,95 \% \mathrm{Cl} 1.30-3.93, \mathrm{p}=0.004)$ and for multiparity $(73.9 \% \mathrm{vs} .85 .6 \%), \mathrm{HR}=2.16$, $95 \% 1.10-4.25, p=0.026$. On multivariate analysis worse DFS remained to be significantly worse for women with early menopause and multiparous women $(p=0.004$ and 0.01 , respectively) together with oncotype $R S(p<0.001)$, see Table 3 . Compared to premenopausal women, postmenopausal women had worse 10-year DFS that approached significance (83.8\% vs. 90.1\%, HR=1.69, 95\% 0.92-3.09, $p=0.09)$. This difference did not remain significant on multivariate analysis $(p=0.289)$. DFS was comparable in the other evaluated subgroups. 
Table 2

DFS and OS and by estrogen exposure

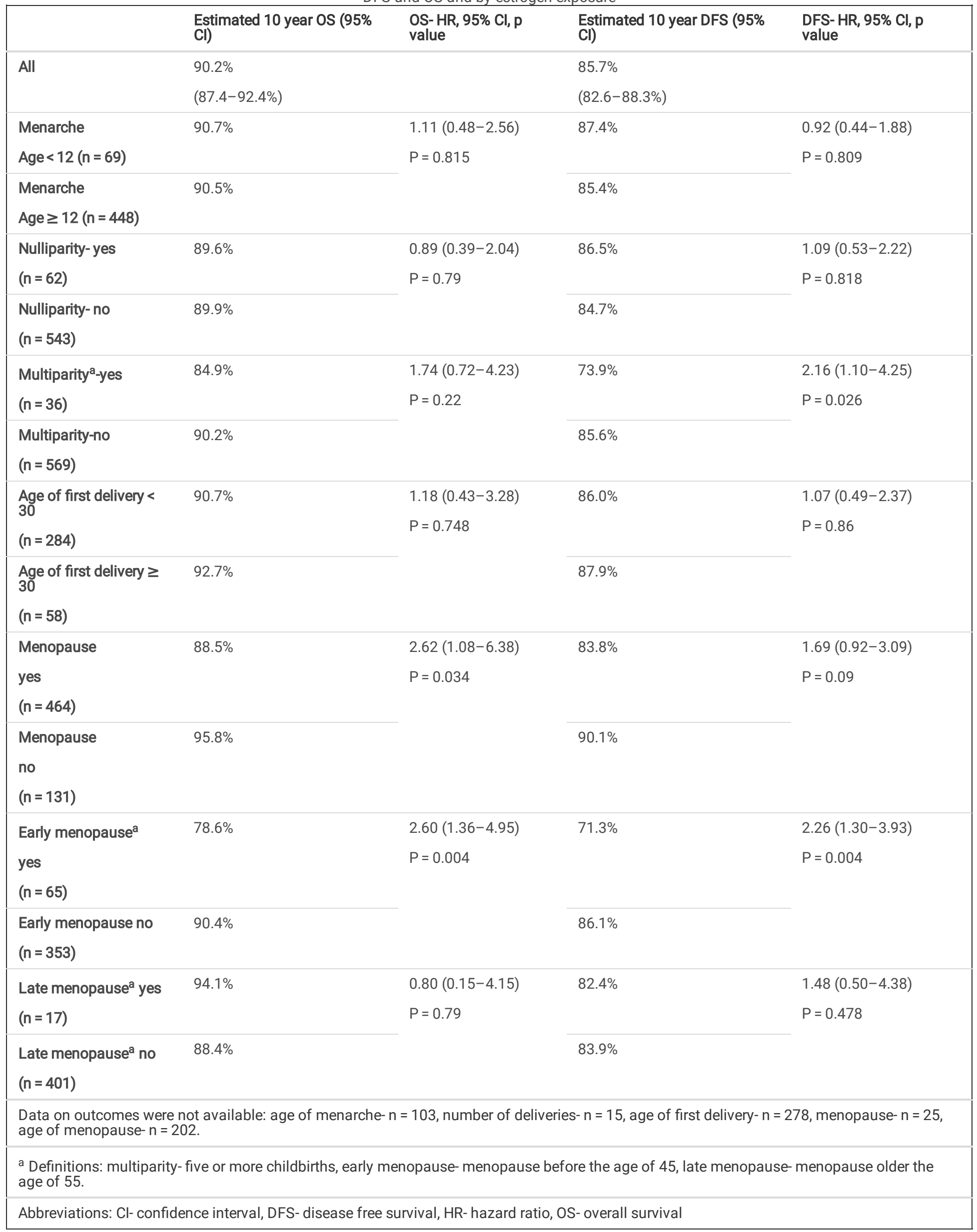


Table 3

Multivariate analysis for OS and DFS

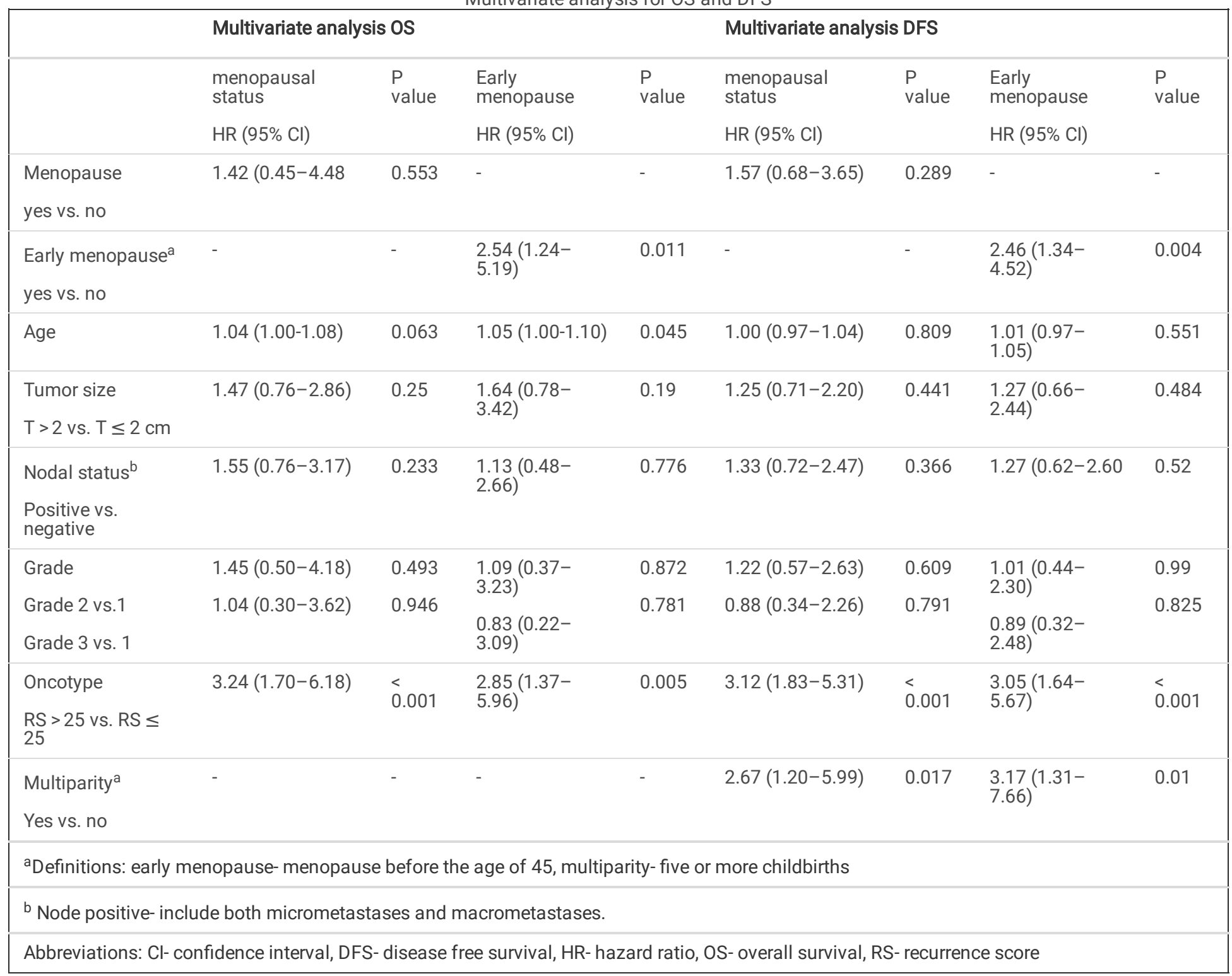

The results of OS are presented in Table 2 and Fig. 2. Women with early menopause had worse 10-year OS compared to women with menopause at later age $(78.6 \%$ vs. $90.4 \%), \mathrm{HR}=2.60,95 \% \mathrm{Cl} 1.36-4.95, \mathrm{p}=0.004$. In multivariate analysis the association between worse 0 S and early menopause remained significant $(p=0.011)$, together with older age $(p=0.011)$ and higher oncotype RS $(p=0.005)$. Compared to premenopausal women estimated 10-year overall survival was significantly worse in post-menopausal women $(88.5 \% \mathrm{vs} .95 .8 \%), \mathrm{HR}=2.62,95 \% \mathrm{Cl} 1.08-6.38, \mathrm{p}=$ 0.034. In multivariate analyses the menopausal status did not remain associated with worse OS ( $p=0.553)$, Oncotype RS was the only variable that was associated with worse OS ( $<<0.001)$, and the impact of older age on worse OS approached significance $(p=0.063)$. Results of the multivariate analyses are shown in Table 3. OS was comparable in the other evaluated subgroups. Of note, administration of chemotherapy for women in high genomic risk was significantly lower in post-menopausal women comparted to pre-menopausal women (70\% vs. $96 \%, p=0.006)$, but for early menopause and multipara women, rates of chemotherapy administration were comparable to their control.

\section{Discussion}

Lifetime exposure to estrogen that is related to reproductive factors including early menarche, late menopause and parity have a well-established impact on breast cancer risk [2]. While the increased risk is more prominent for ER positive disease rather than ER negative disease, few studies address the effect of reproductive factors on other tumor characteristics and outcomes. Here, we investigated the influence of endogenous estrogen exposure on early-stage luminal breast cancer characteristics including also the impact on genomic risk. Overall, the impact of the evaluated endogenous exposures on breast cancer characteristics was relatively limited. Menopause was associated with stronger ER intensity, but with PR negativity. Women with early menarche were more likely to have PR positive disease. Oncotype RS, a well validated prognostic and predictive factor that is not a subject for inter-laboratory heterogeneity [19], was comparable in all of the evaluated subgroups. No other associations between parity, age of first delivery and age of menopause with tumor characteristics were identified. 
Data suggest possible differential effect of reproductive risk factors and obesity according to breast cancer subtype, with parity, age at menarche, age at first birth, breastfeeding and obesity demonstrating stronger associations with luminal subtype compared to other subtypes [17, 20], however data on the effect of these factors of the intensity of ER and PR staining are scarce. In our analysis an association between early menarche and PR positivity was identified. This is in line with other studies showing a higher concordance of ER/PR positive breast cancer in women with early age of menarche [20-22].

In this study, we reported on the influence of endogenous estrogen exposures on outcome with a follow-up duration of more than 10 years. Interestingly, despite comparable tumor characteristics, early menopause and multiparity were each independently associated with worse outcomes, and these differences remained significant in multivariate analysis. Postmenopausal women also had worse OS compared to premenopausal women, but this difference did not remain significant in multivariate analysis and can probably be attributed to non-breast cancer mortality in older population.

Our finding of adverse outcomes of women who menopaused before the age of 45 is meaningful and might have important implication of treatment decisions. Menopause gives rise to cardiovascular morbidity and metabolic syndrome [23, 24]. Earlier age of menopause enhances the risk for cardiovascular morbidity and mortality [25-27]. Given the excellent prognosis of women with luminal early breast cancer, the most common cause of death in this population is cardiovascular disease [28, 29]. Adjuvant treatment for breast cancer might increase cardiovascular morbidity. Anthracycline based adjuvant chemotherapy that are often recommended to women with high genomic risk or to women with high clinical risk [18, 30], might cause cardiotoxicity. Adjuvant treatment with aromatase inhibitors, which are consider the standard of care in all postmenopausal women [31] and can often be considered for higher risk premenopausal in combination with ovarian function suppression [32], have increased odds for cardiovascular morbidity compared to both tamoxifen or placebo [33, 34]. Adjuvant radiation that is given to the vast majority of women after breast conserving surgery, is also associated with cardiovascular toxicity when treating left sided disease [35]. Tailoring treatment decisions based on the individual risk of breast cancer recurrence and on other comorbidities including risk of cardiovascular disease are required. Modifications to reduce treatment related cardiovascular toxicity, such as anthracyclines sparing chemotherapy [36], shorter treatment with Als [33] and omission of radiation or partial breast irradiation [37,38] should be considered. Our findings demonstrated that age of menopause might also be an important factor when weighing the risk and benefit balance of treatment options.

Multiparity was also associated with worse DFS. This finding is significant and is in line with previous publications [39, 40], however due to the low number of multiparous women, the conclusions that can be drawn are limited. Multiparity might be associated with low socioeconomic status [41, 42], and hence lower adherence to treatment and higher risk of comorbidities [43]. Multiparty is also an independent predictor for obesity [44, 45], which may also explain worse outcome after breast cancer diagnosis, both due inferior specific breast cancer survival [46] and non-breast cancer mortality [47]. These hypotheses might partially explain our finding, however, our data on weight and socioeconomic status were insufficient. Larger scales studies are needed to elucidate the effect on multiparity on breast cancer outcome.

We acknowledge the limitations of a single-center retrospective cohort with inherent biases. The histopathological variables were not re-evaluated for the study by central pathology. On the other hand, considering the scarcity of data on the effect of endogenous estrogen on breast cancer characteristics our cohort represents a large number of patients with long duration of follow up. The effect on all known prognostic pathological characteristics were meticulously collected and the assessment of endogenous estrogen impact on Oncotype DX score is novel.

In conclusion, our results suggest little impact of endogenous estrogen exposure on breast cancer characteristics of early stage, luminal disease. Early menopause and multiparity were associated with worse outcome. These findings might be related to other comorbidities or direct influence on breast cancer pathogenesis. Given the impact of early menopause and multiparty on outcome, these variables should be taken into consideration in treatment decisions on early-stage luminal breast cancer.

\section{Declarations}

\section{Conflict of interest}

Dr. Korzets reports speaker honorarium from Roche, outside of submitted work.

Dr. Moore reports speaker honorarium from from Roche and Merck, all outside the submitted work.

Prof. Yerushalmi reports personal fees from: Roche (Consulting, invited speaker), Pfizer (Consulting), Novartis (Consulting), Teva (Invited speaker), Medison (Invited speaker), MSD (Invited speaker), Astra-Zeneca (Invited speaker) and Novartis (Invited speaker), all outside the submitted work.

Dr. Goldvaser reports personal fees from: Roche (honorarium), Pfizer (honorarium), Novartis (honorarium and consulting) and Oncotest (honorarium) all outside the submitted work.

All other authors declare no conflict of interest.

\section{Ethical approval}


All procedures in the study were in accordance with the ethical standards of the institutional ethics committee and with the 1964 Helsinki declaration and its later amendments or comparable ethical standards.

Ethical Review Board: Rabin medical center institutional ethics committee, reference number: 0576-19-RMC

As data were analyzed anonymously, no informed consent was required.

\section{Funding}

None for all authors

\section{Author contribution}

All authors made substantial contributions to conception and design, and/or acquisition of data, and/or analysis and interpretation of data and participated in drafting the article or revising it critically for important intellectual content and gave final approval of the version to be submitted and any revised version.

\section{Data Availability statement}

The datasets generated during and/or analysed during the current study are available from the corresponding author on reasonable request.

\section{References}

1. Siegel RL, Miller KD, Jemal A. Cancer statistics, 2019. CA Cancer J Clin. 2019 Jan;69(1):7-34. https://doi.org/10.3322/caac.21590

2. Key T, Appleby P, Barnes I, Reeves G. Endogenous Hormones and Breast Cancer Collaborative Group. Endogenous sex hormones and breast cancer in postmenopausal women: reanalysis of nine prospective studies. J Natl Cancer Inst. 2002 Apr;94(8):606616. https://doi.org/10.1093/jnci/94.8.606

3. Howlader N, Altekruse SF, Li Cl, Chen VW, Clarke CA, Ries LA, et al. US incidence of breast cancer subtypes defined by joint hormone receptor and HER2 status. J Natl Cancer Inst. 2014 Apr;106(5):dju055. https://doi.org/10.1093/jnci/dju055

4. Collaborative Group on Hormonal Factors in Breast Cancer. Menarche, menopause, and breast cancer risk: individual participant meta-analysis, including 118964 women with breast cancer from 117 epidemiological studies. Lancet Oncol 2012 Nov;13(11):1141-

51. https://doi.org/10.1016/s1470-2045(12)70425-4

5. Ritte R, Lukanova A, Tjønneland A, Olsen A, Overvad K, Mesrine S, et al. Height, age at menarche and risk of hormone receptor-positive and negative breast cancer: a cohort study. Int J Cancer. 2013 Jun;132(11):2619-29. https://doi.org/10.1002/ijc.27913

6. Nichols HB, Schoemaker MJ, Cai J, Xu J, Wright LB, Brook MN, et al. Breast cancer risk after recent childbirth: A Pooled Analysis of 15 Prospective Studies. Ann Intern Med . 2019 Jan;170(1):22-30. https://doi.org/10.7326/m18-1323

7. Albrektsen G, Heuch I, Hansen S, Kvåle G. Breast cancer risk by age at birth, time since birth and time intervals between births: exploring interaction effects. Br J Cancer. 2005 Jan;92(1):167-75. https://doi.org/10.1038/sj.bjc.6602302

8. Rosner B, Colditz GA, Willett WC. Reproductive risk factors in a prospective study of breast cancer: the Nurses' Health Study. Am J Epidemiol. 1994 Apr;139(8):819-35. https://doi.org/10.1093/oxfordjournals.aje.a117079

9. Key TJ, Appleby PN, Reeves GK, Roddam A, Dorgan JF, Longcope C, et al. Endogenous Hormones Breast Cancer Collaborative Group. Body mass index, serum sex hormones, and breast cancer risk in postmenopausal women. J Natl Cancer Inst. 2003 Aug;95(16):1218-26. https://doi.org/10.1093/jnci/djg022

10.

11. Lauby-Secretan B, Scoccianti C, Loomis D, Grosse Y, Bianchini F, Straif K. International Agency for Research on Cancer Handbook Working Group. Body fatness and cancer--Viewpoint of the IARC Working Group. N Engl J Med. 2016 Aug;375(8):794-8. https://doi.org/10.1056/nejmsr1606602

12. Missmer SA, Eliassen AH, Barbieri RL, Hankinson SE. Endogenous estrogen, androgen, and progesterone concentrations and breast cancer risk among postmenopausal women. J Natl Cancer Inst. 2004 Dec;96(24):1856-65. https://doi.org/10.1093/jnci/djh336

13. Farhat GN, Cummings SR, Chlebowski RT, , Parimi N, Cauley JA, Rohan TE, et al. Sex hormone levels and risks of estrogen receptor-negative and estrogen receptor-positive breast cancers. J Natl Cancer Inst. 2011 Feb;103(7):562-70. https://doi.org/10.1093/jnci/djr031

14. Waks AG, Winer EP. Breast cancer treatment: a review. JAMA. 2019 Jan;321(3):288-300. https://doi.org/10.1001/jama.2018.19323

15. Sparano JA, Crager MR, Tang G, Gray RJ, Stemmer SM, Shak S. Development and Validation of a Tool Integrating the 21-Gene Recurrence Score and Clinical-Pathological Features to Individualize Prognosis and Prediction of Chemotherapy Benefit in Early Breast Cancer. J Clin Oncol. 2020 Dec;39(6):557-564. https://ascopubs.org/doi/full/10.1200/JC0.20.03007 
16. Inwald EC, Klinkhammer-Schalke M, Hofstädter F, Zeman F, Koller M, Gerstenhauer M, et al. ki-67 is a prognostic parameter in breast cancer patients: results of a large population-based cohort of a cancer registry. Breast Cancer Res Treat. 2013 Jun;139(2):539-52.

17. Clark GM, McGuire WL, Hubay CA, Pearson OH, Marshall JS. Progesterone receptors as a prognostic factor in stage II breast cancer. N Engl J Med. 1983 Dec;309(22):1343-7. https://doi.org/10.1056/nejm198312013092240

18. Lambertini M, Santoro L, Del Mastro L, Nguyen B, Livraghi L, Ugolini D, et al. Reproductive behaviors and risk of developing breast cancer according to tumor subtype: A systematic review and meta-analysis of epidemiological studies. Cancer Treat Rev. 2016 Sep;49:65-76. https://doi.org/10.1016/j.ctrv.2016.07.006

19. Sparano JA, Gray RJ, Makower DF, Pritchard KI, Albain KS, Hayes DF, et al. Adjuvant chemotherapy guided by a 21-gene expression assay in breast cancer. N Engl J Med . 2018 Jul;379(2):111-121. https://doi.org/10.1056/nejmoa1804710

20. Curtit E, Mansi L, Maisonnette-Escot Y, Sautière JL, Pivot X. Prognostic and predictive indicators in early-stage breast cancer and the role of genomic profiling: Focus on the Oncotype DX ${ }^{\circledR}$ Breast Recurrence Score Assay. Eur J Surg Oncol. 207 may;43(5):921-930. https://doi.org/10.1016/j.ejso.2016.11.016

21. Yang XR, Chang-Claude J, Goode EL, Couch FJ, Nevanlinna H, Milne RL, et al. Associations of breast cancer risk factors with tumor subtypes: a pooled analysis from the Breast Cancer Association Consortium studies. J Natl Cancer Inst. 2011 feb;103(3):250-63. https://doi.org/10.1093/jnci/djq526

22. Huang WY, Newman B, Millikan RC, Schell MJ, Hulka BS, Moorman PG. Hormone-related factors and risk of breast cancer in relation to estrogen receptor and progesterone receptor status. Am J Epidemiol. 2000 Apr;151(7):703-14. https://doi.org/10.1093/oxfordjournals.aje.a010265

23. Yoo KY, Tajima K, Miura S, Takeuchi T, Hirose K, Risch H,et al. Breast cancer risk factors according to combined estrogen and progesterone receptor status: a case-control analysis. Am J Epidemiol. 1997 Aug;146(4):307-14. https://doi.org/10.1093/oxfordjournals.aje.a009271

24. Coyoy A, Guerra-Araiza C, Camacho-Arroyo I. Metabolism regulation by estrogens and their receptors in the central nervous system before and after menopause. Horm Metab Res. 2016 Aug;48(8):489-96.

25. Morselli E, Santos RS, Criollo A Nelson MD, Palmer BF, Clegg DJ. The effects of oestrogens and their receptors on cardiometabolic health. Nat Rev Endocrinol. 2017 Jun;13(6):352-64.

26. Yang L, Lin L, Kartsonaki C, Guo Y, Chen Y, Bian Z et al. Menopause characteristics, total reproductive years, and risk of cardiovascular disease among chinese women. Circ Cardiovasc Qual Outcomes. 2017 Nov;10(11):e004235. https://doi.org/10.1161/CIRCOUTCOMES.117.004235

27. Ley SH, Li Y, Tobias DK, Manson JE, Rosner B, Hu FB, et al. Duration of reproductive life span, age at menarche, and age at menopause are associated with risk of cardiovascular disease in women. J Am Heart Assoc. 2017 Nov; 6(11):e006713. https://doi.org/10.1161/JAHA.117.006713

28. Peters SA, Woodward M. Women's reproductive factors and incident cardiovascular disease in the UK Biobank. Heart. 2018 Jul;104(13):10691075. http://dx.doi.org/10.1136/heartjnl-2017-312289

29. Patnaik JL, Byers T, DiGuiseppi C, Dabelea D, Denberg TD. Cardiovascular disease competes with breast cancer as the leading cause of death for older females diagnosed with breast cancer: a retrospective cohort study. Breast Cancer Res. 2011 Jun;13(3):R64.

30. Yancik R, Wesley MN, Ries LA, Havlik RJ, Edwards BK, Yates JW. Effect of age and comorbidity in postmenopausal breast cancer patients aged 55 years and older. JAMA. 2001 Feb;285(7):885-92.

31. Cardoso F, van't Veer LJ, Bogaerts J, Slaets L, Viale G, Delaloge S, et al. 70-Gene signature as an aid to treatment decisions in early-stage breast cancer. N Engl J Med. 2016 Aug;375(8):717-29. https://doi.org/10.1056/nejmoa1602253

32. Visvanathan K, Fabian CJ, Bantug E, Brewster AM, Davidson NE, DeCensi A, et al. Use of endocrine therapy for breast cancer risk reduction: ASCO clinical practice guideline update. J Clin Oncol. 2019 Nov;37(33):3152-3165. https://doi.org/10.1200/jco.19.01472

33. Francis PA, Regan MM, Fleming GF, Láng I, Ciruelos E, Bellet M,et al. International Breast Cancer Study Group. Adjuvant ovarian suppression in premenopausal breast cancer. N Engl J Med. 2015 Jan;372(5):436-46. https://doi.org/10.1056/nejmoa1412379

34. Goldvaser H, Barnes TA, Šeruga B, Cescon DW, Ocaña A, Ribnikar D, et al. Toxicity of extended adjuvant therapy with aromatase inhibitors in early breast cancer: a systematic review and meta-analysis. J Natl Cancer Inst. 2018 Jan;110(1). https://doi.org/10.1093/jnci/djx141

35. Amir E, Seruga B, Niraula S, Carlsson L, Ocaña A, et al. Toxicity of adjuvant endocrine therapy in postmenopausal breast cancer patients: a systematic review and meta-analysis. J Natl Cancer Inst. 2011 Sep;103(17):1299-309. https://doi.org/10.1093/jnci/djr242

36. Darby SC, Ewertz M, McGale P, Bennet AM, Blom-Goldman U, Brønnum D, et al. Risk of ischemic heart disease in women after radiotherapy for breast cancer. N Engl J Med. 2013 Mar;368(11):987-98.

37. Blum JL, Flynn PJ, Yothers G, Asmar L, Geyer CE Jr, Jacobs SA et al. Anthracyclines in Early Breast Cancer: The ABC Trials-USOR 06-090, NSABP B-46-I/USOR 07132, and NSABP B-49 (NRG Oncology). J Clin Oncol. 2017 Aug ;35(23):2647-

2655. https://doi.org/10.1200/JC0.2016.71.4147

38. Kunkler IH, Williams LJ, Jack WJ, Cameron DA, Dixon JM, et al. PRIME II investigators. Breast-conserving surgery with or without irradiation in women aged 65 years or older with early breast cancer (PRIME II): a randomised controlled trial. Lancet Oncol. 2015 Mar; 16(3):266-

Page 11/14 
73. https://doi.org/10.1016/s1470-2045(14)71221-5

39. Korzets Y, Fyles A, Shepshelovich D, Amir E, Goldvaser H. Toxicity and clinical outcomes of partial breast irradiation compared to whole breast irradiation for early-stage breast cancer: a systematic review and meta-analysis. Breast Cancer Res Treat. 2019 Jun;175(3):531545. https://doi.org/10.1007/s10549-019-05209-9

40. Sun X, Nichols HB, Tse CK, Bell MB, Robinson WR, Sherman ME, et al. Association of parity and time since last birth with breast cancer prognosis by intrinsic subtype. Cancer Epidemiol Biomarkers Prev. 2016 Jan;25(1):60-7. https://doi.org/10.1158/1055-9965.epi-15-0864

41. Jääskeläinen A, Roininen N, Karihtala P, Jukkola A. High parity predicts poor outcomes in patients with luminal B-Like (HER2 Negative) early breast cancer: A Prospective Finnish Single-Center Study. Front Oncol. 2020 Aug;10:1470. https://doi.org/10.3389/fonc.2020.01470

42. Kutty VR, Thankappan KR, Kannan KP, Aravindan KP. How socioeconomic status affects birth and death rates in rural Kerala, India: results of a health study. Int J Health Serv. 1993 ;23(2):373-86. https://doi.org/10.2190\%2F9N4P-F1L2-13HM-CQVW

43. Shawky S, Rashad H, Khadr Z (2018) Reproductive health inequalities in Egypt. Evidence for guiding policies, final report: UNFPA/ASRO.

44. Schultz WM, Kelli HM Varghese T, Shen J, Sandesara P, et al. Socioeconomic Status and cardiovascular outcomes: challenges and interventions. Circulation. 2018 May;137(20):2166-2178. https://doi.org/10.1161/CIRCULATIONAHA.117.029652

45. Heliövaara M, Aromaa A. Parity and obesity. J Epidemiol Community Health. 1981 Sep; 35(3):197-9. https://doi.org/10.1136/jech.35.3.197

46. Hajiahmadi M, Shafi H, Delavar MA. Impact of parity on obesity: a cross-sectional study in Iranian women. Med Princ Pract. 2015 Jan;24(1):7074. https://doi.org/10.1159/000368358

47. Niraula S, Ocana A, Ennis M, Goodwin PJ. Body size and breast cancer prognosis in relation to hormone receptor and menopausal status: a meta-analysis. Breast Cancer Res Treat. 2012 Jul;134(2):769-81. https://doi.org/10.1007/s10549-012-2073-x

48. Dignam JJ, Wieand K, Johnson KA, Raich P, Anderson SJ, Somkin C, et al. Effects of obesity and race on prognosis in lymph node-negative, estrogen receptor-negative breast cancer. Breast Cancer Res Treat. 2006 Jun; 97(3):245-54. https://doi.org/10.1007/s10549-005-9118-3

\section{Figures}



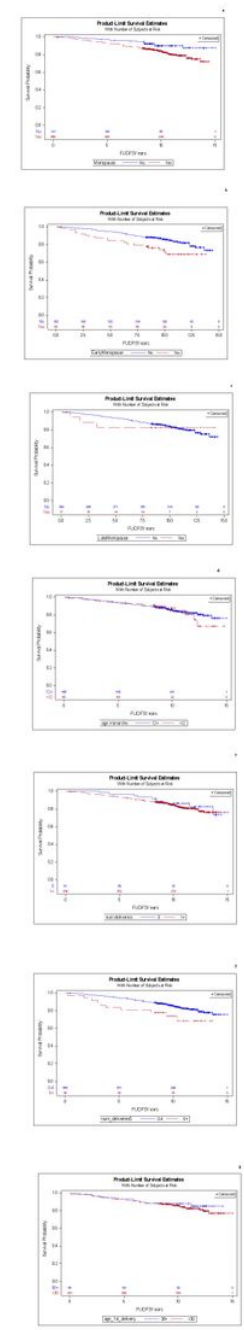

\section{Figure 1}

Disease free survival probabilities in (a) menopausal women, (b) early menopause (menopause before the age of 45), (c) late menopause (menopause older the age of 55), (d) early age at menarche (menarch before the age of 12), (e) nullipara (f) number of deliveries (0-4 Vs. 5 and more), (g) age at fisrt live birth (30 years and older vs. $<30$ years). 

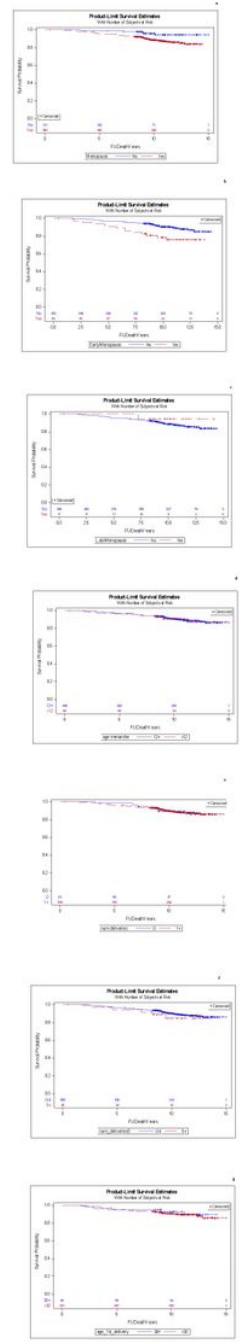

Figure 2

overall survival probabilities in (a) menopausal women, (b) early menopause (menopause before the age of 45), (c) Late menopause (menopause older the age of 55), (d) early age at menarche (menarche before the age of 12), (e) nullipara (f) number of deliveries (0-4 Vs. 5 and more), (g) age at fisrt live birth (30 years and older vs. $<30$ years). 